\title{
Cervical Myelopathy Associated with Deep Neck Muscle Rhabdomyolysis after Polysubstance Abuse: A Case Report
}

\author{
Jari Honkaniemi ${ }^{a}, b, c$ Jaana Rummukainen ${ }^{d} \quad$ Pinne Väänänen $^{e}$ \\ Matti Vuorialho ${ }^{f}$ \\ aDepartment of Neurology, Vaasa Central Hospital, Vaasa, Finland; 'b Department of

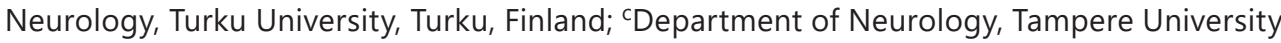 \\ Hospital, Tampere, Finland; 'Department of Clinical Pathology, Kuopio University Hospital, \\ Kuopio, Finland; 'Department of Psychiatry, Kuopio University Hospital, Kuopio, Finland; \\ fDepartment of Radiology, Vaasa Central Hospital, Vaasa, Finland
}

\section{Keywords}

Myelopathy · Spinal cord infarction · Venous hypertension

\begin{abstract}
When used appropriately, buprenorphine and oxycodone are safe drugs. They are, however, widely abused in combination with other drugs. Here we describe a case series of 8 patients with cervical myelopathy and rhabdomyolysis of the adjacent deep neck muscles after using an opioid in combination with other drugs. All patients were young males who had a previous history of polysubstance abuse. Six of the patients had used buprenorphine in combination with pregabalin and/ or benzodiazepines, and one patient had used oxycodone with pregabalin and/or benzodiazepines. One patient used buprenorphine with amphetamine. After taking the drugs, they all reported feeling drowsy and then falling asleep. On waking, they noticed weakness in their extremities. However, only one patient woke with his head in a flexed position. A varying degree of tetraparesis was observed. Cerebrospinal fluid analysis revealed elevated protein levels and white blood cell count. Blood creatine kinase was elevated in 7 patients. Spinal cord MRI showed a hyperintense spinal lesion at the level of $\mathrm{C} 1$ - Th3 vertebrae associated with rhabdomyolysis in the adjacent, paravertebral deep neck muscles. We suggest that polysubstance abuse, especially the combination of an opioid with another drug with GABA-agonistic properties, caused a compartment syndrome of the deep paravertebral muscles without excessive head flexion. This subsequently led to compression of the external vascular structures, resulting in venous congestive myelopathy.
\end{abstract}




\section{Introduction}

When used appropriately, buprenorphine and oxycodone are generally safe drugs without any serious adverse effects. In Scandinavia, however, they are commonly abused in combination with benzodiazepines and pregabalin. Here we describe eight patients with a combination of myelopathy and rhabdomyolysis of the adjacent deep muscles of the neck after taking an opioid together with other drugs.

\section{Case Presentation}

The 8 patients were treated in three Finnish hospitals. The authors evaluated all the medical records, MRI data, and laboratory results of the patients. The MRI studies were made using 1.5-3 T machines, depending on the hospital in question. Imaging results were re-evaluated by a neuroradiologist (M.V.).

All patients were men around their 20's. Six of the patients reported using buprenorphine intravenously and one intranasally (Table 1). One patient had presumably used oxycodone intravenously. Seven patients had also used several other drugs, particularly pregabalin and/or benzodiazepines. One patient used amphetamine together with buprenorphine. All patients reported feeling drowsy and then falling asleep after taking the drugs. However, none of the patients reported passing out immediately. After waking up, they noticed limb weakness and difficulty in walking. One patient recollected waking up with his head flexed.

In the emergency department, examination of the patients revealed varying degrees of tetraparesis. When examined, the patients had absent or depressed deep tendon reflexes in lower limbs and negative Babinski sign apparently due to spinal shock. A common feature in lower limb muscle strength was no movement at all or traces of contraction. Except for patient \#7 (Table 1), the serum creatine kinase levels of the patients were elevated. Otherwise, the patients' blood tests were unremarkable and other organ involvement was not observed. One patient was positive for hepatitis C. The cerebrospinal fluid analysis showed both an elevated protein level and white blood cell count. Sagittal T2-weighted MRI scans of the spinal cords revealed extensive hyperintense lesions of the cervical spinal cord (shown in Fig. 1). These lesions consisted of most of the cervical spinal cord which, in some cases, extended to the upper thoracic region. Axial T2-weighted images of the spinal cord showed peripheral hypointensity. Asymmetric hyperintense lesions in fluid-sensitive sequences were usually seen in the paravertebral deep neck muscles, mainly in the multifidus and semispinalis cervicis and capitis muscles (shown in Fig. 1). The muscle fasciae were thickened and, when investigated, showed contrast enhancement. Limb muscles were not investigated.

Patients were treated with steroids, hypertonic saline, acyclovir, and various antibiotics without response. None of the patients developed renal failure. Patient \#6 was operated on 2 days after onset of the symptoms. Laminectomy was performed at the C3 to C6 levels. The muscle fascia was clearly thickened and, after performing an incision to the fascia, the swollen and compressed muscle bulged out. Histological samples taken from the fascia and muscle showed myxotic and thickened fibrosis in the soft tissue. Focal necrotic fibers and very mild lymphocytic inflammation were seen in the skeletal muscle (shown in Fig. 2).

Patients \#1, \#4, \#5, and \#6 continued to abuse buprenorphine after being discharged from hospital. Patient \#3 had oxycodone in his medication after the incident. Patients \#1, \#4, and \#7 later died, and no autopsy was performed.

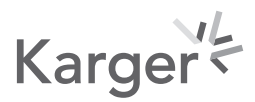




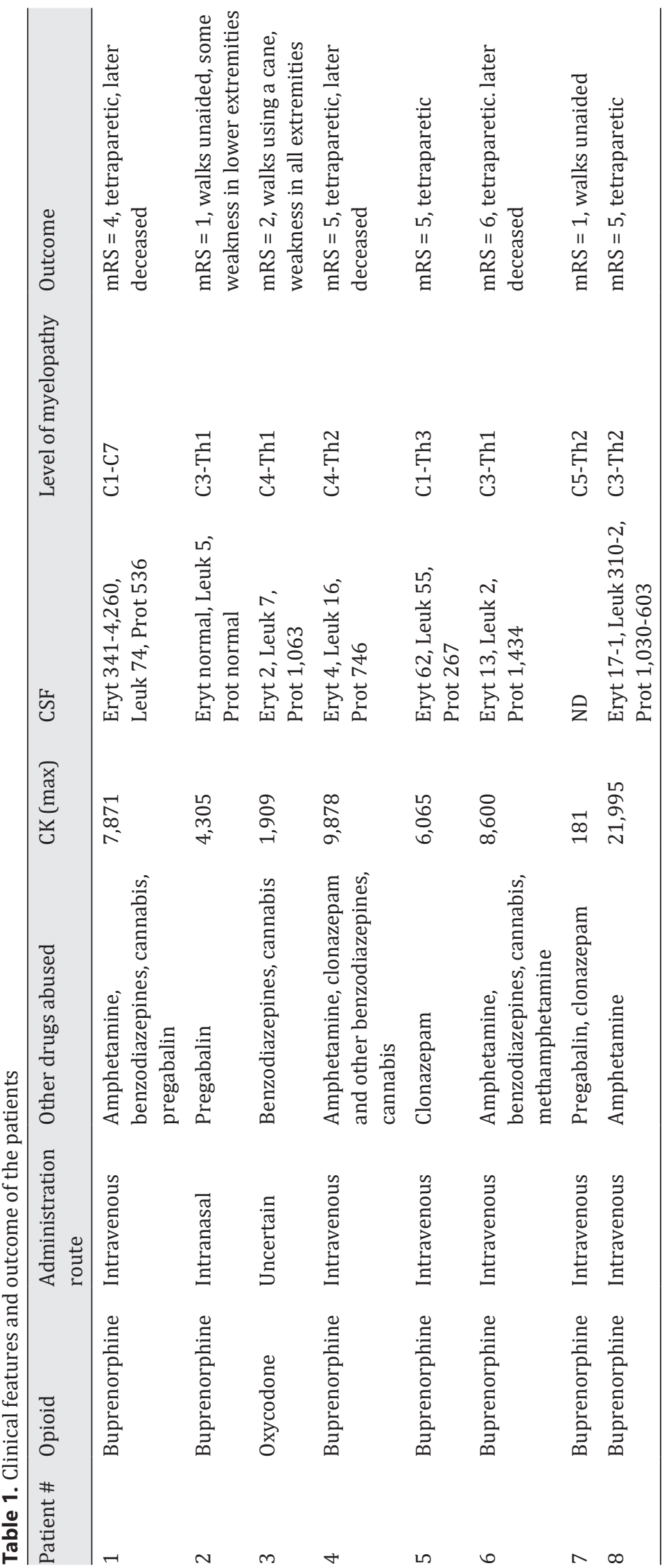

Karger' 

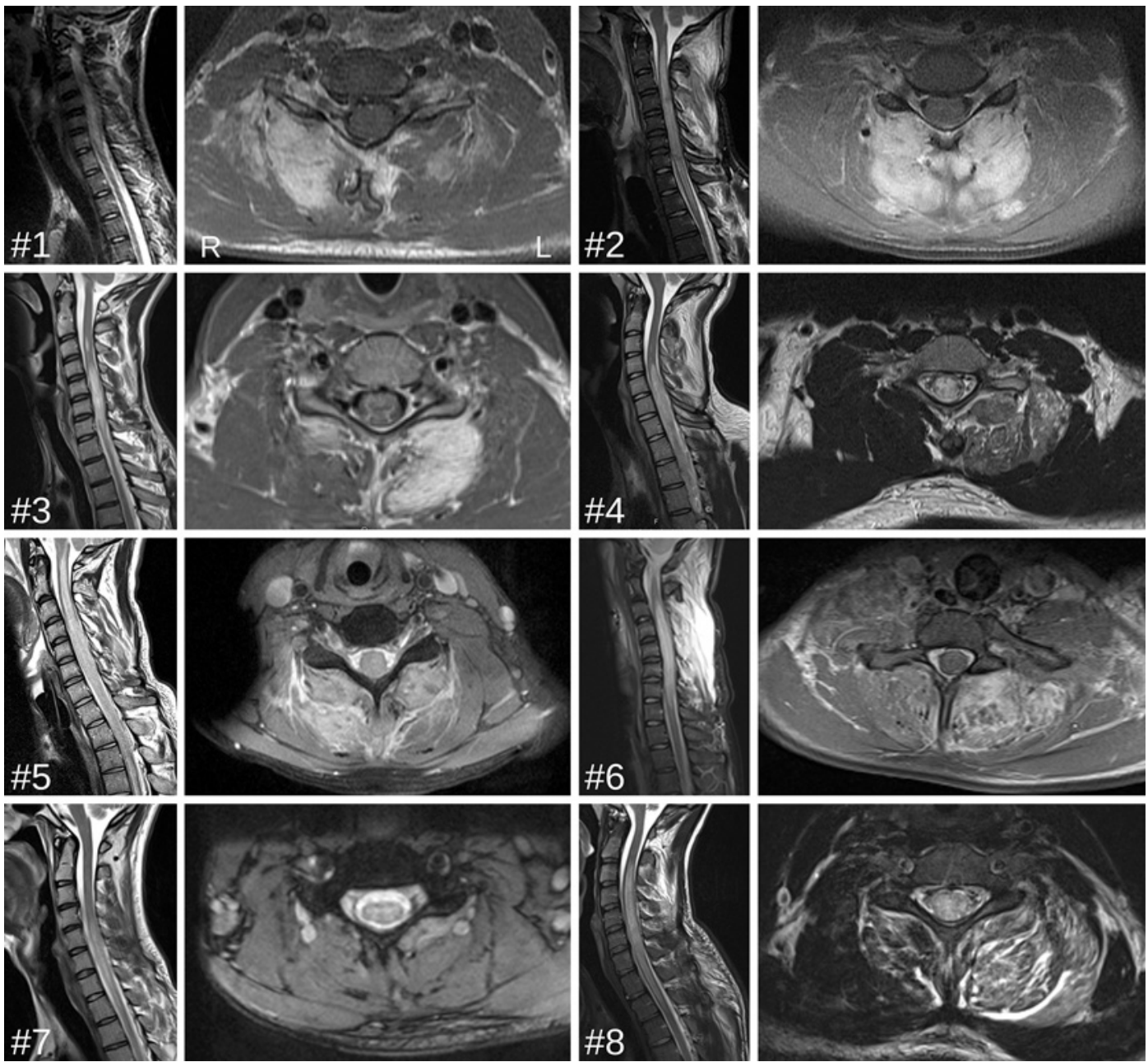

Fig. 1. Sagittal and axial MRI images of the cervical spinal cord. T2-and T2*-weighted images demonstrating hyperintense lesions on the cervical medulla and the adjacent multifidus and splenic cervicis/capitis muscles. A hypointense rim surrounds the central hyperintense lesion in the axial images of the spinal cord of patient \#7.
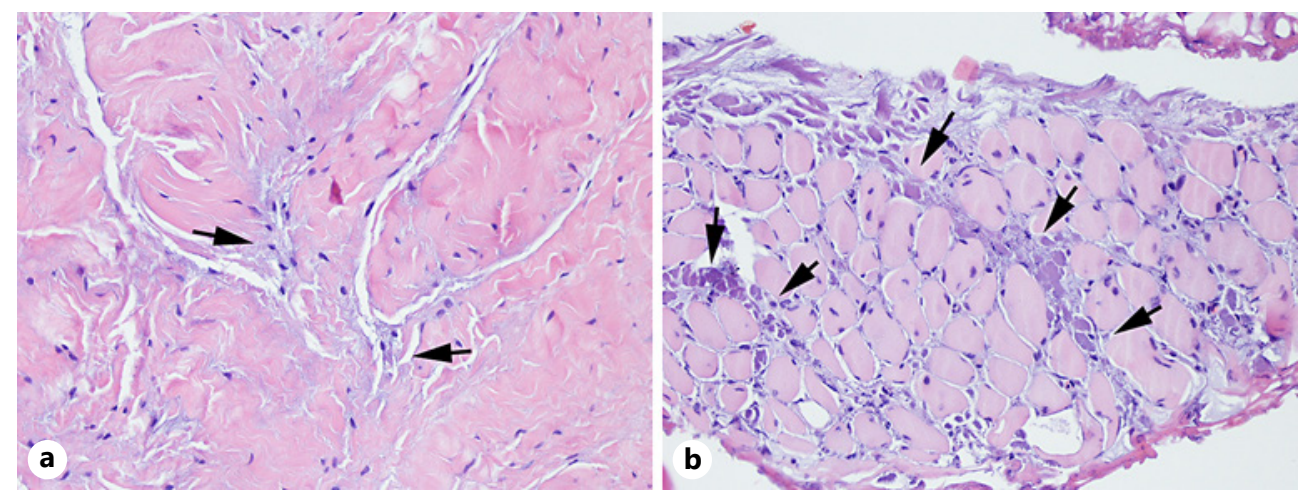

Fig. 2. Histological samples obtained from patient \#6. a Myxotic fibrosis in soft tissue between thickened collagen fibers (arrows; hematoxylin and eosin, $\times 200$ ). b Necrotic fibers in between small rounded skeletal muscle fibers (arrows; hematoxylin and eosin, $\times 200$ ). 


\section{Discussion}

Several striking similarities were observed in the cases presented here. First, the patients were all young males around their 20's. Despite their young age, however, they all had a clear history of polysubstance abuse. Second, they all reported feeling drowsy and falling asleep after taking the drugs. Third, the myelopathy was always in the cervical region, and it was associated with rhabdomyolysis of the adjacent paravertebral deep neck muscles. Finally, all patients had taken a combination of a synthetic opioid with other drugs before developing the myelopathy.

Midcervical flexion myelopathy resulting in quadriplegia is a well-known complication of surgical procedures requiring patients to be in flexed cervical spine position [1-3]. Intoxication and substance abuse have caused similar complications [4-7]. In previous single case reports describing drug-induced myelopathy, the patients had used heroin and oxycodone, but not buprenorphine. It has been suggested that heroin-induced myelopathy is a neuroimmunological process [4]. In our patients, this seems unlikely, as five of the patients presented here were treated with either an opioid or continued using them without relapse. Furthermore, we found no signs of intrathecal IgG synthesis in the CSF.

Hirayama disease, also known as flexion myelopathy, is a slowly progressing amyotrophy in the upper extremities. To separate Hirayama disease from the acute myelopathy following neck flexion, it has been proposed that the term subacute flexion myelopathy should be used [8]. Because patients have usually had their head in a flexed position [5-13], it has been suggested that neck flexion causes focal compression or stretching of the cervical cord, leading to spinal cord infarction via compromised microvascular perfusion [3]. In some subacute flexion myelopathy cases, the lesions shown in MRI scans were rather small [2, 9] compared to the extensive lesions of the patients reported here. In addition, rhabdomyolysis was not always reported $[2,9]$ and when present, it was not necessarily associated with the MRI changes in the deep cervical muscles $[4,6]$.

Myelopathy associated with adjacent deep neck muscle rhabdomyolysis has been described in five single case reports after surgery performed in flexed position [8], after oxycodone use [10], and after intoxication [5, 11, 12]. Interestingly, only one of the patients described here recollected waking up with his head in a flexed position, suggesting that excessive head flexion is not essential for developing myelopathy. This is supported by previous findings that intoxication and surgery may cause subacute cervical myelopathy without extensive hyperflexion of the neck $[2,4,13]$.

Both heroin and buprenorphine use have been shown to cause rhabdomyolysis via compartment syndrome in the extremities. The hypothesis of a compartment syndrome in the neck muscles is supported by MRI findings that show thickened fascia around swollen muscles and observations during the operation on patient \#6. Opioid-induced compartment syndrome in the extremities has several causes, including paravenous injections, direct muscle compression, and infections associated with intramuscular injections. The etiology of the compartment syndrome reported here is not, however, caused by any of these factors. Direct muscle compression is not a causative factor, as neck muscle rhabdomyolysis caused by compression affects variable superficial muscles in the face and neck. These muscles were, however, spared in all the patients presented here. We suggest therefore that lying in the same position for a prolonged period after taking the drugs resulted in in a compartment syndrome and rhabdomyolysis of the deep neck muscles without any excessive neck flexion.

Since both the myelopathy and rhabdomyolysis were adjacent in the cervical region, we suspect a causal relationship between the two. Because swollen muscles are likely to compress the adjacent vascular structures, ischemic spinal cord infarction is possible. However, since

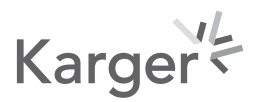


the anterior and posterior spinal cord arteries originate from the vertebral arteries, they are not affected by the compression. Therefore, damage to the arterial circulation of the spinal cord would be limited to the compromised circulation via the segmental arteries. A more likely reason for the spinal cord damage is impaired venous flow of the spinal cord, leading to venous hypertensive myelopathy. This hypothesis is supported by the finding of the hypointense peripheral rim surrounding the central hyperintensity in T2-weighted images (shown in Fig. 1), which is a specific sign of venous hypertensive myelopathy [14]. Thus, we suggest that polysubstance abuse produced a compartment syndrome of the deep paravertebral neck muscles which, in turn, impaired the venous flow of the spinal cord, resulting in venous hypertensive myelopathy. Fasciotomy would therefore be the treatment of choice. This operation was performed on patient \#6 without any response, suggesting that if this process develops for long enough, the damage is irreversible.

Buprenorphine is a safe drug for treating opiate addiction. The 2007 background document reviewing the safety of buprenorphine, methadone and naltrexone for treating opioid dependence stated that there is no reason to believe that buprenorphine is commonly associated with any serious adverse effects [15]. This document formed the basis of the WHO guidelines for the psychosocially assisted pharmacological treatment of opioid dependence, where no mention of the interaction between buprenorphine and, e.g., pregabalin was made. We conclude therefore that physicians treating opioid-dependent patients with buprenorphine and those patients using these drugs with a tendency to polysubstance abuse should be aware of the interaction between buprenorphine and other drugs and the devastating long-term sequelae described here. This diagnosis carries a poor prognosis since three of the eight patients presented here died within a few years of the incident.

\section{Acknowledgments}

Drs. Ulrich Tacke, Tuukka Puolakka, and Eerika Koskinen are thanked for their contribution in the acquisition of data.

\section{Statement of Ethics}

This study protocol was reviewed and approved by the administrative medical directors of the hospital districts of Vaasa, Kuopio, and Tampere University Hospitals, Approval Numbers $\S 61$ and $\S 118 / 2021$. This study has been granted an exemption from requiring written informed consent and ethics approval was not required by the medical directors of the Vaasa Central Hospital and Kuopio University Hospital and the research manager of the Tampere University Research Services. In Finland, legislation covering the secondary use of health and social data means that the customer and register data created during health and social service sector activities will be used for purposes other than the primary reason for which they were originally saved.

\section{Conflict of Interest Statement}

Matti Vuorialho and Jaana Rummukainen have no conflicts of interest to declare. Pinne Väänänen has received funding for travel expenses from Indivior; Jari Honkaniemi has received funding for travel expenses and/or speaker honoraria from Orion Pharma, Teva, Bayer, Biogen, GSK, Abbvie, Boehringer Mannheim, and Pfizer.

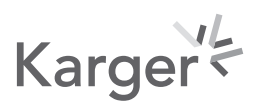




\section{Funding Sources}

Study Funded by the State Research Funding of Vaasa Hospital District based on the Health Care Act (EVO; 1326/2010).

\section{Author Contributions}

Jari Honkaniemi: Drafting/revision of the manuscript for content, including medical writing for content; major role in the acquisition of data; analysis and interpretation of data. Jaana Rummukainen: Drafting/revision of the manuscript for content, including medical writing for content; analysis and interpretation of data. Pinne Väänänen: Drafting/revision of the manuscript for content, including medical writing for content; analysis and interpretation of data. Matti Vuorialho: Drafting/revision of the manuscript for content, including medical writing for content; analysis or interpretation of data.

\section{Data Availability Statement}

All data generated or analyzed during this study are included in this article. Further enquiries can be directed to the corresponding author.

\section{References}

1 Yahanda AT, Chicoine MR. Paralysis caused by spinal cord injury after posterior fossa surgery: a systematic review. World Neurosurg. 2020;139:151-7.

2 Windfuhr JP, Dülks A. Spinal cord infarction following cricotracheal resection. Int J Pediatr Otorhinolaryngol. 2010;74(9):1085-8.

3 Martínez-Lage JF, Almagro MJ, Izura V, Serrano C, Ruiz-Espejo AM, Sánchez-Del-Rincón I. Cervical spinal cord infarction after posterior fossa surgery: a case-based update. Childs Nerv Syst. 2009;25(12):1541-6.

4 Gupta A, Khaira A, Lata S, Agarwal SK, Tiwari SC. Rhabdomyolysis, acute kidney injury and transverse myelitis due to naive heroin exposure. Saudi J Kidney Dis Transpl. 2011;22(6):1223-5.

5 Kaye KL, Ramsay D, Young GB. Cervical flexion myelopathy after valproic acid overdose. Spine. 2001;26(19): E459-62.

6 Sahni V, Garg D, Garg S, Agarwal SK, Singh NP. Unusual complications of heroin abuse: transverse myelitis, rhabdomyolysis, compartment syndrome, and ARF. Clin Toxicol. 2008;46(2):153-5.

7 Richter RW, Pearson J, Bruun B, Challenor YB, Brust JC, Baden MM. Neurological complications of addiction to heroin. Bull N Y Acad Med. 1973;49(1):3-21.

8 Fehre KS, Weber MA, Hensel C, Weidner N. Tetraparesis as clinical correlate of subacute cervical flexion myelopathy. J Spinal Cord Med. 2016;39(3):359-62.

9 Haisa T, Kondo T. Midcervical flexion myelopathy after posterior fossa surgery in the sitting position: case report. Neurosurgery. 1996;38(4):819-2.

10 Alahmadi H, Zadeh G. Spinal cord injury after prolonged neck flexion, is it an underestimated risk? Can J Neurol Sci. 2011;38(5):794-6.

11 Nielsen AS, Damek DM. Window of opportunity: flexion myelopathy after drug overdose. J Emerg Med. 2012; 42:36-9.

12 Wang TS, Grunch BH, Moreno JR, Bagley CA, Gottfried ON. Drug overdose resulting in quadriplegia. Eur Spine J. 2012;21(4):S521-4.

13 Morandi X, Riffaud L, Amlashi SF, Brassier G. Extensive spinal cord infarction after posterior fossa surgery in the sitting position: case report. Neurosurgery. 2004;54(6):1512-6.

14 Hurst RW, Grossman RI. Peripheral spinal cord hypointensity on T2-weighted MR images: a reliable imaging sign of venous hypertensive myelopathy. AJNR Am J Neuroradiol. 2000;21:781-6.

15 Gray A. Systematic review of the safety of buprenorphine, methadone and naltrexone. Guidelines for the psychosocially assisted pharmacological treatment of opioid dependence. Geneva: World Health Organization; 2009. 